\title{
Serum TK1 is a more reliable marker than CEA and AFP for cancer screening in a study of 56,286 people
}

\author{
Yu Wang ${ }^{\mathrm{a}, *}$, Xiaorong Jiang ${ }^{\mathrm{a}}$, Shaoliang Dong ${ }^{\mathrm{b}}$, Jiankun Shen ${ }^{\mathrm{b}}$, Haixia $\mathrm{Yu}^{\mathrm{a}}$, Ji Zhou ${ }^{\mathrm{c}}$, Jin $\mathrm{Li}^{\mathrm{c}, \mathrm{d}}$, \\ Hongbo $\mathrm{Ma}^{\mathrm{c}}$, Ellen $\mathrm{He}^{\mathrm{c}}$ and Sven Skog ${ }^{\mathrm{c}, *}$ \\ ${ }^{a}$ Health Management Center of PLA 180 Hospital, Quanzhou, Fujian, China \\ ${ }^{\mathrm{b}}$ Medicine Management Department, PLA 180 Hospital, Quanzhou, Fujian, China \\ ${ }^{\mathrm{c}}$ Sino-Swed Molecular Bio-Medicine Research Institute, Shanghai, China \\ ${ }^{\mathrm{d}}$ Department of Ophthalmology, Shenzhen People's Hospital, The Second Clinical Medical College of Jinan \\ University, Shenzhen, Guangdong, China
}

\begin{abstract}
.
BACKGROUND: The World Health Organization (WHO) has estimated that the number of cancer patients will increase by about $70 \%$ during the next 25 years world-wide. To deal with this problem, WHO has suggested a focus on prevention of tumor incidence and health screening for early detection of people with tumors.

OBJECTIVE: To investigate the use of thymidine kinase 1 (TK1), CEA and AFP in serum to discover people with malignant tumors through health cancer screening.

METHODS: Of a cohort in 486,085 people of a routine health screening at the Health Centre, Fujun 180 Hospital, Quanzhou city, China, 56,286 people were investigated according to the presence of cancer during 2009-2014. The concentration of CEA and AFP were determined by an electrochemiluminescence immunoassay from Roche Diagnostics e601GmbH and STK1 by a commercial kit based on an enhanced chemiluminescent dot blot assay.

RESULTS: The cancer incident rate increased from $0.048 / 100,000$ to $0.220 / 100,000$. The most common types of tumors were those of the liver, cervix and lung. STK1 correlated to tumor growth rate, was more sensitive than CEA and AFP for discovering people with malignant tumors and more sensitive among people who had diagnosis of malignant tumor. STK1 was also a prognostic biomarker for death at 10-40 months follow-up, while CEA and AFP were not. A combination of these markers increased the sensitivity by about $30 \%$.
\end{abstract}

CONCLUSION: STK1 is a reliable biomarker for discovering people with malignant tumors in cancer screening.

Keywords: Thymidine kinase 1 (TK1), CEA, AFP, health screening

\section{Introduction}

Cancer has become one of the major diseases among the hazards of modern human life. China's third ret-

${ }^{*}$ Corresponding authors: Sven Skog, Sino-Swed Molecular BioMedicine Research Institute, Shanghai 299333, China. Tel: +86 46736402152; Fax: +86 75526031330; E-mail: svenisak@icloud. com; Yu Wang, Health Management Center of PLA 180 Hospital, Quanzhou 362000, Fujian, China. Tel.: +86 133313835652; Fax: +86 59522761738; E-mail: wy652@sina.com. rospective survey report on causes of deaths (20042005) showed that chronic non-infectious disease was the leading cause of death of residents, accounting for $82.5 \%$ of all causes of death, with cancer accounting for $22.3 \%$ [1]. As reported by the World Health Organization (WHO) in 2014, the cancer incident rate world-wide is expected to increases by about $70 \%$ during the next 20-25 years, if lifestyles continue as they are today [2]. Data from the China Cancer Registration Centre confirm this assumption. The incidence rate of cancer increased from 184.81/100.000 to 
286.69/100.000 during 1989-2008, with higher incident rate of cancer in men compared to women [3].

Cancer is a chronic disease of abnormal proliferating cells. To deal with the uncontrolled increase in the cancer incident rate, WHO has suggested focusing on prevention and early detection of tumors. The imaging techniques used in oncology have improved extensively during the last decades, but still they are unable to discover small-size, early-stage of tumors. In an effort overcome this limitation, researchers have been testing several serum tumor markers and biomarkers for over more than 60 years, also with limited results [4].

Thymidine kinase 1 (TK1) is an enzyme in the salvage pathway of DNA synthesis and is closely related to proliferating cells. TK1 has been studied extensively since it's discovery in the beginning of 1950 s, with more than 3,000 scientific publications, covering knowledge from TK1 gene properties to the protein structure of TK1. Briefly, TK1 concentration/activity is low in the G1 stage of the cell cycle, increases in $\mathrm{S}$-phase/G2 stages and degrade in the mitotic stage, showing it's close relation to rate of cell proliferation [5-8]. In 2002, the authors developed a novel chicken $\operatorname{IgY}$ antibody raised against the C-terminal end of the human thymidine kinase 1 . This antibody (pI 8.3) recognized both the $25 \mathrm{kDa}$ subunit and the tetrameric form of human TK1. The TK1 antibody inhibited the catalytic activity of the enzyme [9]. In native Western blot analysis of TK1 in serum (STK1) from pre-treated patients with gastric cancer $[9,10]$, non-Hodgkin's lymphoma [11], and breast cancer [12], one single band corresponding to human TK1 was obtained, demonstrating the high specificity of the antiTK1 IgY antibody. The weak TK1 band found in serum of healthy individuals showed the high sensitivity of the IgY TK1 antibody in serum of cancer patients [9-12]. This IgY TK1 antibody has been used in the clinical setting in health screening and oncology application for more than a decade [13-21]. STK1 combined with different conventional imaging techniques and biopsy/histopathology as well as serum tumor related-biomarkers in cancer screening will hopefully limit the increases of death from cancer in the future. There are projects in China today to support such an idea at health centres [15-18,20,21]. CEA and AFP are produced in normal cell and at a high level in the presence of a cancer [4], but are not directly part of the growth "machinery" [22]. Serum CEA is found in some types of cancer diseases, for example colorectal, medullary thyroid and metastatic breast cancer, while serum AFP is mainly related to primary hepatocellular cancer [4].

In this study the imaging techniques were combined with the serum markers of TK1, CEA and AFP in order to improve cancer screening. This is the first retrospective investigation of cancer incidence in Quanzhou city, Fujian province, China. This study is based on 56,286 people out of a cohort of 486,085 persons, who participated in a health screening at Fujun 180 Hospital, Quanzhou, during 2009-2014.

\section{Patients and methods}

\subsection{Patients}

Routine cancer screening was done on 486,085 persons (285,805 men and 200,280 women) at the Health Centre, Fujun 180 Hospital, Quanzhou, China, during 2009-2014. The main cancer diseases in this area are liver, cervix and lung cancer. Cancer screening is an important part of the Health Management Center. The people undergoing the health-screening test were covered by medical insurance. This health center serves people from both inside of Quanzhou city and from the rural area outside. The age distribution was 13-86 years, mean age $44.8 \pm 11.8$ years, representing young students; people working in administration, schools, hospitals; farmers, and so on. Quanzhou is located in the southeast of China. To discover cancer or suspected cancer diseases different medical test protocols were used including traditional radiography, conventional imaging examination combined with blood and urine tests and physical examination. In addition, tumor-associated markers such as TK1, CEA and AFP were used. Presences of malignancies were confirmed by histopathology.

In a group of people ( $n=56,286$ : 38,816 men, 17,362 women, of the cohort of 486,085 ; mean age $45.7 \pm 10.9$ years, range $21-85$ years) TK1, CEA and AFP were also analysed in the serum to see how well these markers discover cancer patients. Since this study was based on clinical routine and not on a clinical trial, the tumor/biomarkers investigations were performed on people who voluntarily chose to be tested for tumor markers. All serum samples were collected in the morning before food intake. Blood collected without anticoagulant was centrifuged at $800 \times \mathrm{g}$ for $10 \mathrm{~min}$, two to three hours after collection. The serum samples were analysed for TK1, CEA and AFP directly or stored at $-20^{\circ} \mathrm{C}$. 


\subsection{Follow-up}

The follow-up was performed on 52 patients (mean age 55.1 years, range $28-85)$ of a group of patients with malignant tumors $(n=89)$ between 10 and 40 months. The follow-up was closed 13 October 2015. Thirty-seven patients were not included as they could not be contacted. The time of surgery and dead/alive status were included in the follow-up.

\subsection{TK1 assay in serum}

The high specific anti-TK1 IgY antibody raised against a C-terminal peptide of the human TK1 (residue 195-225, GQPAG PDNKE NCPVP GKPGE AVAAR KLFAPQ) was assembled into a commercial kit. The concentration of STK1 was measured by using this kit, based on an enhanced chemiluminescence (ECL) dot blot assay (SSTK Ltd., Shenzhen, China). Samples comprising $3 \mu \mathrm{l}$ of serum were directly applied to nitrocellulose membrane. The serum samples were probed with the TK1 chicken IgY antibody raised against a peptide of TK1. The immunogenic TK1 peptide was dotted at different concentrations (2.2, 6.6 and 20 picomolar (pM) as an extrapolation standard. The intensities of the spots on the membrane were determined by a CCD camera (CIS-1 Imaging System, SSTK Ltd., Shenzhen, China). From the intensities of the TK1 standard of known concentrations, the STK1 concentration was calculated and expressed as pM. The coefficient of variatiowas less than $10 \%$. The reference threshold value of STK1 was $0.5 \mathrm{pM}$ corresponding to STK1 mean value of healthy persons with no or very low frequency of tumor diseases or tumor-related diseases. Cancer patients with STK1 values of $\leqslant 0.5$ $\mathrm{pM}$ were regarded as having a better prognoses compared to patients with cancer patients with STK1 values $>0.5 \mathrm{pM}$.

\subsection{CEA and AFP assays}

CEA and AFP levels were performed by electrochemiluminescence immunoassay (Roche Diagnostics e601GmbH, Mannheim, Germany) in which the reference cut-off values were $5.0 \mathrm{ng} / \mathrm{ml}$ and $10 \mathrm{ng} / \mathrm{ml}$, respectively. The value was considered positive or negative for the marker when the level was above or below the cut-off value. The electrochemiluminescence automatic immunoassay analyzer is based on paramagnetic particles as a solid phase, biotin-streptavidin-detection system and two-dimensional bar code technology. It is a highly sensitive light detection system that provides excellent low-end sensitivity and broad dynamic measuring ranges.

\subsection{Statistical analysis}

Chi-square test and p-test were applied for comparison of STK1, CEA and AFP expressions (SPSS17.0, UK). Student's t-test was used to compared patients below and above the cut-off values of the serum markers. A p-value of less than 0.05 was regarded as statistically significant. This study was conducted in accordance with the Helsinki Declaration of 1983 and was approved by the ethics committee of Fujun 180 Hospital, Quanzhou, (No. LL2009003).

\section{Results}

\subsection{Frequency of tumors}

Twenty-four different types of tumors were analyzed (Table 1). The three most frequent tumors were those of the liver, >cervix, >and lung. The cancer incident rate of all type of tumors together increased by 4.5 times from 0.048 in 2009 to 0.220 in 2014 (Table 2). While the incident rates of cervix, liver, lung, kidney, stomach, colorectal and breast cancer increased, the incident rates for esophagus, leukemia and thyroid cancer were almost unchanged during the six years period (Table 2).

\subsection{Cancer incidence in relation to age}

Malignant tumors were determined in 56,286 persons of the 486,085 people participating in the health screening, randomly collected. The number of people with malignancies found in the group of 56,285 people was 89 . These persons were divided into four age groups: $13-20,21-40,41-60$ and $>61$ years old. No person with a malignant tumor was found in the group of people 13-20 years old. In the groups of people $21-40,41-60$ and $>60$ years old, the number of persons with malignant tumors were 7,57 and 25 , respectively. The cancer incidence rate increased by age from 0.032 in the group of people $21-40$ years old up to 0.44 among people above 60 years of age (Fig. 1).

\subsection{Sensitivity of STK1,CEA and AFP in relation to age}

The ability of STK1, CEA and AFP in serum to detect people with malignant tumors was determined in 56,286 of the 486,085 people participating the health screening, randomly collected. The group of people 
Table 1

Number of persons with malignant tumors in the time period from 2009 to 2014

\begin{tabular}{lccccccc}
\hline Type & 2009 & 2010 & 2011 & 2012 & 2013 & 2014 & Total \\
\hline Number investigated & 31,087 & 65,127 & 82,796 & 91,180 & 106,460 & 103,435 & 486,085 \\
Liver & 10 & 21 & 34 & 36 & 48 & 51 & 200 \\
Cervical & 0 & 15 & 26 & 22 & 45 & 55 & 163 \\
Lung & 2 & 8 & 26 & 31 & 28 & 44 & 139 \\
Renal & 0 & 2 & 5 & 12 & 19 & 18 & 56 \\
Esophageal & 0 & 6 & 8 & 10 & 18 & 11 & 53 \\
Stomach & 2 & 4 & 7 & 9 & 12 & 12 & 46 \\
Colorectal & 1 & 1 & 0 & 3 & 6 & 9 & 20 \\
Breast & 0 & 1 & 1 & 2 & 5 & 7 & 16 \\
Leukemia & 0 & 1 & 3 & 4 & 2 & 1 & 11 \\
Thyroid & 0 & 0 & 1 & 2 & 5 & 1 & 9 \\
Bladder & 0 & 0 & 0 & 1 & 1 & 4 & 6 \\
Pelvic & 0 & 1 & 0 & 0 & 3 & 1 & 5 \\
Brain & 0 & 2 & 0 & 0 & 0 & 2 & 4 \\
Lymphoma & 0 & 0 & 0 & 0 & 2 & 1 & 3 \\
Aplastic anemia & 0 & 0 & 0 & 0 & 1 & 2 & 3 \\
Nasopharynx & 0 & 0 & 0 & 2 & 0 & 0 & 2 \\
Abdominal stromal tumors with metastasis & 0 & 0 & 1 & 0 & 0 & 1 & 2 \\
Bile & 0 & 0 & 0 & 0 & 0 & 2 & 2 \\
Anterior mediastinum & 0 & 0 & 1 & 1 & 0 & 0 & 2 \\
Retroperitoneal & 0 & 0 & 0 & 0 & 1 & 0 & 1 \\
Thymus & 0 & 0 & 0 & 0 & 0 & 1 & 1 \\
Pharynx & 0 & 0 & 0 & 0 & 0 & 1 & 1 \\
Throat & 0 & 0 & 0 & 1 & 0 & 0 & 1 \\
Glioma & 0 & 0 & 0 & 0 & 0 & 0 & 0 \\
Total & 15 & 62 & 113 & 136 & 196 & 224 & 746 \\
\hline
\end{tabular}

Table 2

Cancer incident rates of different types of tumors 2009-2014. Total number of people investigated during this period was 482,085

\begin{tabular}{cccccccccccc}
\hline Years & Overall & Cervical & Liver & Lung & Kidney & Esophagus & Stomach & Breast & Colorectal & Leukemia & Thyroid \\
\hline 2009 & 0.048 & 0 & 0.032 & 0.006 & 0 & 0 & 0.006 & 0 & 0.003 & 0 & 0 \\
2010 & 0.095 & 0.057 & 0.032 & 0.012 & 0.003 & 0.009 & 0.006 & 0.004 & 0.002 & 0.002 & 0 \\
2011 & 0.138 & 0.076 & 0.041 & 0.031 & 0.006 & 0.01 & 0.008 & 0.003 & 0 & 0.004 & 0.001 \\
2012 & 0.141 & 0.056 & 0.037 & 0.032 & 0.012 & 0.01 & 0.009 & 0.005 & 0.003 & 0.004 & 0.002 \\
2013 & 0.185 & 0.101 & 0.045 & 0.026 & 0.018 & 0.017 & 0.011 & 0.011 & 0.006 & 0.002 & 0.005 \\
2014 & 0.22 & 0.126 & 0.049 & 0.043 & 0.017 & 0.011 & 0.012 & 0.016 & 0.009 & 0.001 & 0.001 \\
Total & 0.155 & 0.081 & 0.041 & 0.029 & 0.012 & 0.011 & 0.009 & 0.008 & 0.004 & 0.002 & 0.002 \\
\hline
\end{tabular}

Table 3

Number of patients with malignant tumors above cut-off values (positive rate) of STK1, CEA and AFP at different ages. All types of tumors are considered together. The values in parents are percentage values

\begin{tabular}{lcccc}
\hline Type & $13-20$ years & $21-40$ years & $41-60$ years & $>60$ years \\
\hline STK1 & 0 & $4 / 7(57.1)$ & $31 / 57(54.4)$ & $14 / 25(56.0)$ \\
CEA & 0 & 0 & $11 / 57(19.6)$ & $14 / 25(56.0)$ \\
AFP & 0 & $2 / 7(28.6)$ & $14 / 57(24.6)$ & $4 / 25(16.0)$ \\
\hline
\end{tabular}

with malignancies $(n=89)$ was divided into four age groups: $13-20,21-40,41-60$ and $>61$ years old. The cut-off value of STK1 was $0.5 \mathrm{pM}$, CEA $5.0 \mathrm{ng} / \mathrm{ml}$ and AFP $10 \mathrm{ng} / \mathrm{ml}$. All types of tumors were considered together. The sensitivity of STK1, CEA and AFP among the people with age-specific diagnosis of malignancy is shown in Table 3.

STK1 showed higher sensitivity in all age groups compared to CEA and AFP, except for the age group $>60$ years, where the sensitivities of STK1 and CEA were almost the same. A combination of STK1 with CEA and AFP increased the sensitivity by about $20 \%$, however not statistically significant (Table 4).

The sensitive of STK1, CEA and AFP of individual tumor types was also analyzed (Table 5). The number of patients above the cut-off value of STK1 was $\geqslant 50 \%$ in six of the seven types of tumors studied, but only in two types of tumors for CEA (colorectal, lung). In the case of AFP there were no tumor types $\geqslant 50 \%$. Liver cancer patients reached $48.3 \%$ above 
Table 4

Percentage of patients with malignant tumors above cut-off values (positive rate) in combination of STK1, CEA and AFP (STK1 + $\mathrm{X})$. The positive rates were calculated on all types of cancer patients together

\begin{tabular}{lcll}
\hline Type & $\%$ & $\mathrm{X}^{2}$ & $\mathrm{p}^{*}$ \\
\hline STK1 & 54.2 & & \\
STK1+AFP & 62.6 & 0.41 & 0.522 \\
STK1+CEA & 67.3 & 0.94 & 0.332 \\
STK1+CEA+AFP & 72.3 & 1.8 & 0.179 \\
\hline
\end{tabular}

* Chi-square test.

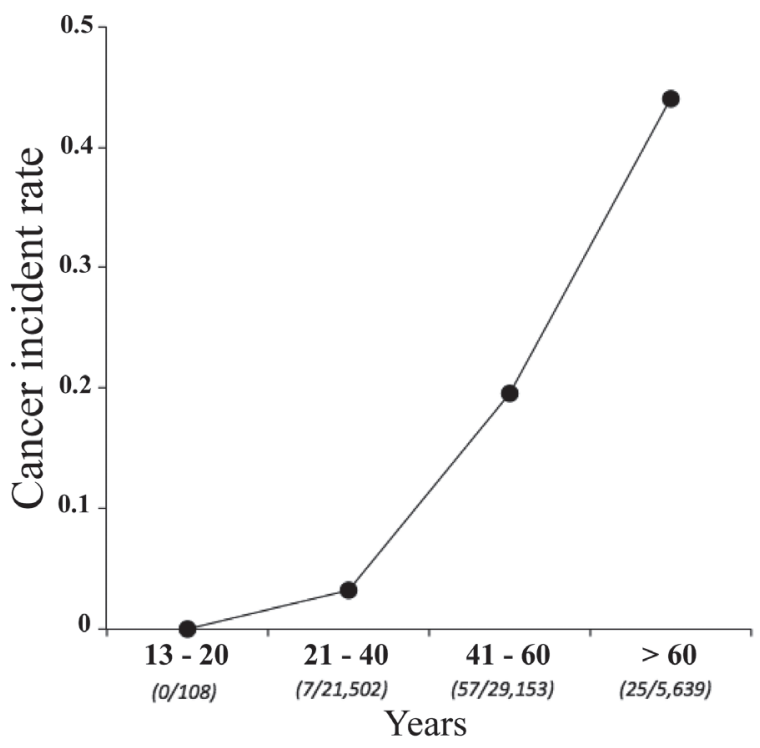

Fig. 1. Cancer incident rate in relation to ages. The values in the brackets are the number of people with malignant tumors in relation to the total number of people investigated.

cut-off value of AFP and lung cancer patients reached $12.5 \%$, but no patients with the other type of tumors showed AFP above the cut-off values. Thus, AFP is specific for liver malignancy. STK1 is more sensitive in patients with stomach cancer $(100 \%)$ compared to patients with cervix cancer $(47.4 \%)$. A combination of STK1 and CEA increased the number of patients above the cut-off value in colorectal, lung and liver cancer patients, while a combination of STK1 and AFP increased the number of patients above cut-off value of liver cancer (Table 5).

There was no correlation between the values of STK1/CEA, STK1/AFP and CEA/AFP (Fig. 2).

\subsection{Sensitivity of STK1,CEA and AFP in relation to survival}

Of the 89 persons with malignant diseases, 52 patients could be followed-up for 10 to 40 months.
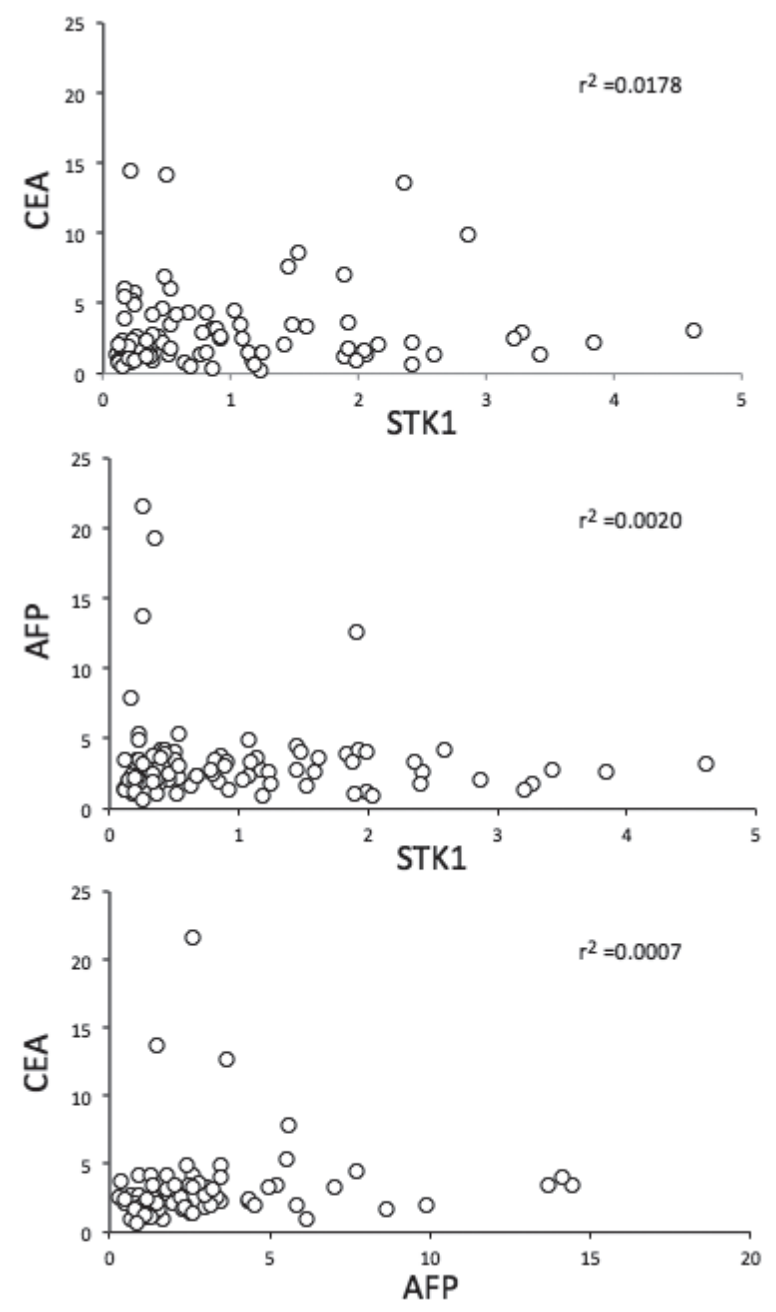

Fig. 2. Correlation between values of STK1/CEA, STK1/AFP and CEA/AFP.

All types of cancer patients were considered together. There were a significantly higher number of dead patients in the STK1 group above the cut-off value compared to below the STK1 cut-off value. The patients with high STK1 values were statistically significant older compared to the patients with low STK1. There were no significantly differences between the number of dead and living patients below and above the cut-off values of CEA and AFP (Table 6).

\section{Discussion}

The rank of the 10 most common malignancies in China is lung, stomach, liver, breast, oesophageal, colon, colorectal, uterine, leukaemia and brain/ nerve [3]. However, since there is a great geographi- 
Table 5

Percentage of patients with malignant tumors above cut-off values (positive rate) of single markers of STK1, CEA and AFP and in combination of these markers $(\mathrm{STK} 1+\mathrm{X})$. The increases in the values of the combinations found were not statistically significant

\begin{tabular}{lrrrrr}
\hline Type & STK1 & CEA & AFP & STK1+CEA & STK1+AFP \\
\hline Stomac & 100.0 & 0.0 & 0.0 & 100.0 & $\mathbf{1 0 0 . 0}$ \\
Colorectal & 83.0 & 60.0 & 0.0 & 80.0 & 83.3 \\
Kidney & 80.0 & 20.0 & 0.0 & $\mathbf{8 1 . 3}$ & 80.0 \\
Lung & 56.0 & 63.5 & 42.5 & $\mathbf{7 3 . 3}$ & 56.0 \\
Liver & 50.0 & 26.6 & 0.0 & 50.0 & $\mathbf{8 2 . 8}$ \\
Esophagus & 50.0 & 16.6 & 0.0 & 47.4 & 50.0 \\
Cervical & 47.4 & 5.3 & & 47.4 \\
\hline
\end{tabular}

Table 6

Number of dead/living patients below and above the cut-off values of STK1, CEA and AFP after 10-40 months follow-up. The test of STK1, CEA and AFP were done on the same patients. The statistical test of the age distribution was done by Student's t-test, while the statistical test of the number of dead/living patients was done by Chi-square test

\begin{tabular}{ccccc}
\hline Type & Age in years & $\mathrm{p}$ & Dead/living & $\mathrm{X}^{2}$ \\
\hline STK1 & & & & \\
Below & $51,5(36-71)$ & & $4 / 20(20.0 \%)$ & \\
Above & $59,0(28-85)$ & $\mathbf{0 , 0 4 9}$ & $13 / 15(86.7 \%)$ & 6,84 \\
CEA & & & $13 / 28(46.4 \%)$ & $\mathbf{0 , 0 0 9}$ \\
Below & $53,8(28-83)$ & 0,245 & $4 / 6(66.7 \%)$ & 0,25 \\
Above & $60,6(42-85)$ & & & \\
AFP & & & $12 / 28(42.9 \%)$ & \\
Below & $54,7(28-85)$ & 0,835 & $5 / 6(83.3 \%)$ & 0,618 \\
Above & $53,7(36-83)$ & & 0,336 \\
\hline
\end{tabular}

cal span, an uneven economic development, and differences of natural environments influencing lifestyle and eating habits, the types of cancer diseases are quit different in different parts of China. Even within local areas of China, that is, provinces, there are significantly different types of cancers. For example, in the Fujian province in the southeast of China, in which this study was performed, in the coastal area the major five cancers are stomach, lung, oesophageal, liver and cervical, while in the northwest mountainous areas the major five types are lung, stomach, liver, colon and breast cancer [23]. In Changle city of Fujian province, which is closely to the area in the present study, lung cancer was reported 2009 to be the most common type of cancer. The general cancer incident rate in Fujian province 2011 was 269.12/100,000 [23].

In this study from the Health Centre of Quanzhou Tumor Hospital located in the southeast part of China the major types were liver, cervical and lung cancer. The incident rate of cancer increased rapidly from 2009 to 2014, by about five times. Quanzhou city developed markedly during this period and the type of tumors found at higher frequency reflects a modern type of lifestyle. Modern and large cities in China today have cancer incident rates between 200/100,000 and 400/100,000 people. Thus, the type of cancer found in Quanzhou city and the cancer incident rates are in agreement with similar studies in this part of China [23].
The main point of this study was to find out whether STK1 could be used to discover people with malignant tumors of different growth rates and how well it works in relation to other commonly used tumorrelated biomarkers, for example, CEA or AFP. It is well known that STK1 is a proliferation biomarker and reflects the growth rate of both normal cells and different type of tumor cells, depending on whether the tumor grows fast or slowly [15-21]. In a study of 444 cancer patients it was shown that the STK1 level was 3 -folds higher in stomach cancer patients compared to patients with cervix cancer [24]. It is known that stomach tumor grow faster than cervix tumor. The results in the present study support a finding that STK1 reflect tumors with higher growth rates, that is, the number of patients with STK1 above the cut-off value (positive rate, see Table 5) was higher in stomach cancer patients $(100 \%)$ compared to patients with cervix cancer $(47.4 \%)$. CEA and AFP reflect different properties of tumor cells, but did not directly survey the tumor growth rate [22], indicated in the present study by the lack of correlation between these markers. Furthermore, CEA and AFP may not be elevated in the presence of some diseases or cancers, especially in early stages of the disease and therefore CEA and AFP are still not recommended for screening of cancer diseases [4].

In the present study, the group of patients with cancer-diagnoses, STK1 showed higher positive rates, 
compared to CEA and AFP. A combination of these markers, particular the combination STK1+CEA+AFP, increased the sensitivity, by about $20 \%$, but did not reach to significance. The lower sensitivity of CEA and AFP compare to STK1 is due to the fact that STK1 is a general proliferation marker covering almost all type of tumors, while CEA and AFP are related only to some type tumors [4]. In particular, AFP is mainly related to liver carcinoma (4). Therefore we also analysed the sensitivity of the markers in individual types of malignancies. Although AFP is specific for liver cancer, STK1 reached the same sensitivity as AFP and could be used as a marker also in patients with liver cancer. The sensitivity of CEA was high in colorectal and lung cancer to the same level as STK1. Since cervix cancer is one of the major tumor disease in this region of China, it is of interest to note that STK1 is more useful for patients with cervix cancer than CEA (positive rate $47.4 \%$ for STK1 compared to $5.3 \%$ for CEA). The combination of CEA with STK1 had benefit for colorectal, lung and liver cancer patient, while a combination of AFP with STK1 increased the sensitivity for patients with liver cancer. STK1 was also found to be prognostic biomarker for the survival of cancer patients, while CEA and AFP were not, which further support the benefit of STK1 over CEA and AFP.

Thus, to obtain information from as many types of tumors as possible in cancer screening, STK1 is to be recommended. The combination of different types of markers is also useful since they reflect different properties of tumor cells.

We conclude that STK1 is more sensitive than CEA and AFP in cancer screening. However, we recommend a combination of different types of tumor- and biomarkers, and different types of imaging, since they reflect different properties of tumor cells, which could be of importance for assessment of the tumor proliferating rate and the prognosis of cancer patients.

\section{Acknowledgements}

This study was made possible through grants from the Health Management Centre of PLA 180 Hospital, Quanzhou, China. We also thank the Sino-Swed Tongkang Biotech. Ltd., Shenzhen, for providing technical support.

\section{References}

[1] W. Chen, Estimation of cancer incidence and mortality in China in 2004-2005, Chinese J Oncol 31 (2009), 664-668.
[2] B.W. Stewart and Ch.P. Wild, World Cancer Report 2014, ISBN 978-92-832-0429-9.

[3] W. Chen, R. Zheng, H. Zeng et al., Trend analysis and projection of cancer incidence in China between 1989 and 2008, Chinese J Oncol 34 (2012), 517-524.

[4] Cigna, Tumor markers for cancer in Cigna medical coverage policy, 1/15/(2015), 1-5.

[5] J.L. Sherley and T.J. Kelly, Regulation of human thymidine kinase during the cell cycle, J Biol Chem 263 (1988), 83508358.

[6] A. Al-Madhoun, W. Tjarks and S. Eriksson, The role of thymidine kinases in the activation of pyrimidine nucleoside analogues, Mini Rev Med Chem 4 (2004), 341-350.

[7] F. Gasparri, N. Wang, S. Skog, A. Galvani and S. Eriksson, Thymidine kinase 1 expression defines an activated G1 state of the cell cycle as revealed with site-specific antibodies and ArrayScan assays, Eur J Cell Biol 88 (2009), 779-785.

[8] Y.L. Chen, S. Eriksson and Z.F. Chang, Regulation and functional contribution of thymidine kinase 1 in repair of DNA damage, J Biol Chem 285 (2010), 27327-27335.

[9] C.J. Wu, R.J. Yang, J. Zhou, S. Bao, L. Zou, Y.R. Mao, Q. He, Production and characterisation of a novel chicken IgY antibody raised against C-terminal peptide from human thymidine kinase 1, J Immuno Method, 277 (2003), 157-169.

[10] L. Zou, P.G. Zhang, S. Zou, Y. Li, Q. He, The half-life of cytosolic thymidine kinase in serum by ECL dot bolt potential marker for monitoring the response to surgery of patients with gastric cancer, Int J Biolog Marker, 17 (2002), 135-140.

[11] Z.L. Pan, X.Y. Ji, Y.M. Shi, J. Zhou, E. He, S. Skog, Serum thymidine kinase 1 concentration as a prognostic factor of chemotherapy-treated non-Hodgkin's lymphoma patients. $J$ Cancer Res Clin Oncol, 136 (2010), 1193-1199.

[12] F.Y. Chen, L.L. Tang, T. Xia, E. He, G.Z. Hu, Y. Li, M. Zhan, J. Zhou, S. Eriksson, S. Skog, Serum thymidine kinase 1 levels predict cancer.free survival following neoadjuvant, surgical and adjuvant treatment of patients with locally advanced breast cancer, Molecular and Clinical Oncology 1 (2013), 894-902.

[13] O. Topolcan and L. Holubec, The role of thymidine kinase in cancer diseases. Expert Opin Med Diagn 2 (2008), 129-141.

[14] S. Aufderklamm, T. Todenhöfer, G. Gakis, S. Kruck, J. Hennenlotter, A. Stenzl and C. Schwentner, Thymidine kinase and cancer monitoring, Cancer Lett 316 (2012), 6-10.

[15] G.Q. Zhang, S.C. Jiao and X.F. Wei, Serum thymidine kinase 1: A proliferation marker for neoplasms in oncologic monitoring of elderly people, J Modern Oncol 16 (2008), 831-832.

[16] X.H. Xu, Y.M. Zhang, X.H. Shu, Z.W. Wang, Y.L. Zhou, H.K. Wen, F. He, E. He, S. Skog, Serum thymidine kinase 1 reflects the progression of premalignant and malignant tumors during therapy, Molecular Medicine Reports 1 (2008), 705-712.

[17] Z.H. Chen, H. Zhou, N.B. Tian et al., Serological thymidine kinase 1 (STK1) indicates an elevated risk for development of malignant tumours, Anticancer Res 28 (2008), 3897-3907.

[18] X.H. Zhang, J.R. Yang, X.Y. Fu, H.X. Liu, X. Yun, B. Song, M. Zhang, E. He and S. Skog, The significance of serum thymidine kinase 1 for the risk screening of cancer development in pre-cancerous diseases, Chin J Health Manag 4 (2008), 35-38.

[19] S.Q. Huang, J. Lin, N. Guo, M. Zhang, X. Yun, S. Liu, J. Zhou, E. He and S. Skog, Elevated serum thymidine kinase 1 predicts risk of pre/early cancerous progression, Asian Pac J Cancer Prev 12 (2011), 497-505.

[20] Z.H. Chen, S.Q. Huang, Y. Wang, A.Z. Yang, J. Wen, X.H. Xu, Y. Chen, Q.B. Chen, Y.H. Wang, E. Hee, J. Zhou and S. 
Skog, Serological thymidine kinase 1 is a biomarker for early detection of tumours - a health screening study on 35,365 people, using a sensitive chemiluminescent dot blot assay, Sensors 11 (2011), 1106411080.

[21] J. Zhou, E. He and S. Skog, The proliferation marker thymidine kinase 1 in clinical use (Review), Mol Clin Oncol 1 (2013), 18-28.

[22] J.T. Wu, Circulating Tumor Markers of the New Millennium.
AACC Press, New York, (2002), pp. 115-117.

[23] Chinese Cancer Registration Annual Report, (2011), National Cancer Center, Disease Prevention and Control Bureau, Ministry of China.

[24] Z.J. Zhang, Y.P. Zheng, Y.F. Lin, X.Q. Mei, Clinical detecting application of TK1 in the diagnosis of common malignant tumors, Int. J. Lab. Med. 35 (2014), 2636-2637. 